\title{
Dyke-Davidoff-Masson Syndrome in the Philippines: A Case Series
}

\author{
Christine C. Sia and Mina N. Astejada \\ Department of Neurosciences, College of Medicine and Philippine General Hospital, University of the Philippines Manila
}

\begin{abstract}
Dyke-Davidoff-Masson Syndrome (DDMS) is a rare condition characterized by seizures, contralateral hemiparesis or hemiplegia and mental retardation. The radiological features are cerebral hemiatrophy and associated compensatory bony alterations. The etiology of this syndrome may be classified as congenital or primary and acquired or secondary. We report four cases of Dyke-Davidoff-Masson Syndrome in the Philippines.
\end{abstract}

Key Words: Dyke-Davidoff-Masson Syndrome, hemiatrophy, hemiplegia, seizure, case series

\section{Introduction}

Dyke-Davidoff-Masson Syndrome (DDMS) is a rare condition characterized by cerebral hypoplasia with homolateral hypertrophy of the skull and sinuses, in association with clinical findings of contralateral hemiparesis, seizures, and mental retardation. Since its discovery, only 23 observations were reported in the literature with no known reported case in the Philippines. The dearth in comprehensive medical reports makes for an astounding statistic considering that it has been more than 75 years since the first reported case. Supplemental reports are clearly necessary and desirable for more insight on this particular disease entity.

\section{Case Presentation}

\section{CASE 1}

A man aged 70 years from Albay presented with complaints of uncontrolled seizures. He was born via normal spontaneous delivery as the second child among seven siblings. At age 6, he was noted to have right-sided hemiparesis and was diagnosed to have poliomyelitis. At age 29, he started to have generalized tonic-clonic seizures, which was poorly controlled with phenytoin. There was epilepsy in his family history and he had a brother with a

Poster presented at the Philippine Neurological Association Annual Research Forum, November 2011, Edsa Shangri-la Hotel, Mandaluyong City (Best Poster).

Corresponding author: Christine C. Sia, MD

Department of Neurosciences

Philippine General Hospital

University of the Philippines Manila

Taft Avenue, Ermita, Manila 1000 Philippines

Telefax No.: +632 5548462

Email: tyne012@yahoo.com similar clinical presentation. On physical examination, atrophy of the right side of the body was noted (Figure 1A). Diffuse slowing was detected by electroencephalography. Plain Cranial CT scan revealed left cerebral atrophy with dilatation of the ipsilateral lateral ventricle and increased prominence of the cerebral sulci (Figures 1B, C).
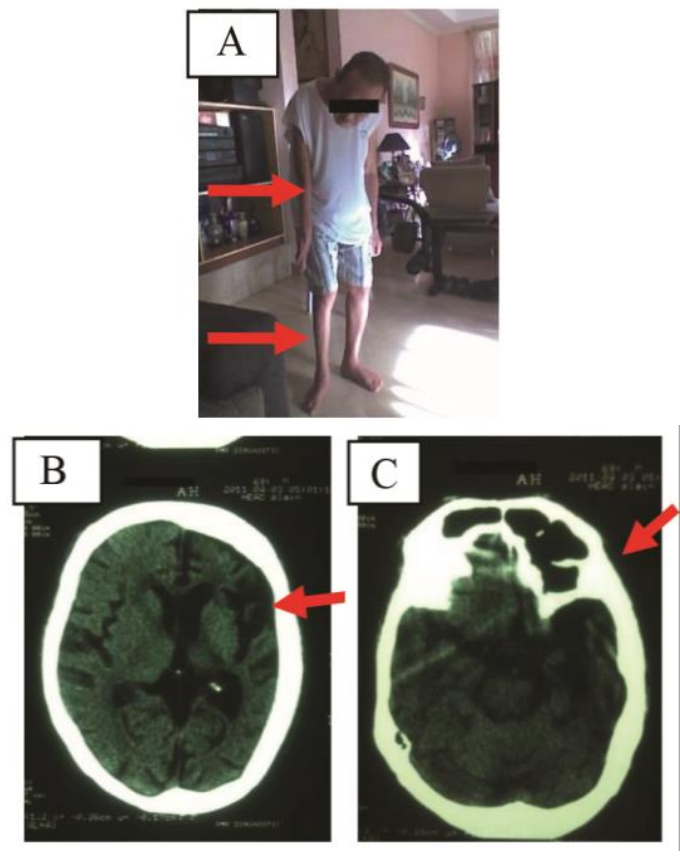

Figure 1. A. Atrophy of the right extremities. B. Cranial CT scan showed increased prominence of the left cerebral sulci with smaller left cerebral hemisphere and ipsilateral ventricular enlargement. C. Increased thickness of the left cranial vault is also visualized.

\section{CASE 2}

A 51-year-old male from Albay, born via normal spontaneous delivery as the youngest child among seven siblings was evaluated for recurrent seizures. Developmental milestones were at par with age until 5 months of age when he started to have generalized tonicclonic seizures. Since then, he developed mental retardation and spastic right hemiparesis. There was epilepsy in his family history, and he had a brother with similar clinical presentation. Physical examination showed spastic hemiparesis and hyperactive deep tendon reflexes on the right extremities (Figure 2A). Electroencephalogram 
revealed a mild diffuse left hemispheric dysfunction as well as multifocal and independent epileptiform activities over the left temporal and right parietotemporal regions. Cranial CT showed left cerebral hemiatrophy with associated thickening of the calvarium (Figures 2B, C).
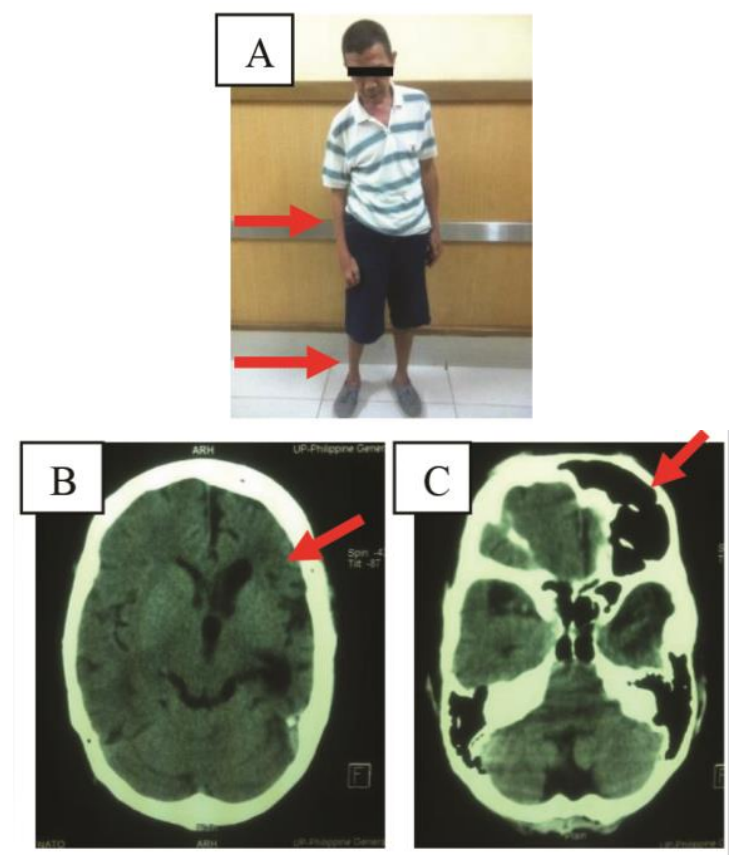

Figure 2. A. Patient with right-sided hemiatrophy. B. Cranial CT showed diffuse atrophy of the left cerebral hemisphere with associated dilatation of the $3^{\text {rd }}$ and left lateral ventricle. C. The left aspect of the calvarium is thickened with hyperplastic left frontal sinus.

\section{CASE 3}

A man aged 20 years from Cavite presented with complaints of seizures for the past 17 years. The patient was born prematurely due to a motor vehicular accident suffered by his mother in his 8th month of gestation. He had a twin brother who died as a result of the event. At birth, he was noted to have a deformed left arm and leg. He also had motor delay thereafter. On examination, he had facial asymmetry with left-sided hemiatrophy and hemiparesis (Figure 3A). An electroenchephalogram revealed slowing of background activity more on the right hemisphere and presence of sharp wave discharges more frequently on the right and occasionally on the left hemisphere. Cranial CT done showed a smaller right cerebral hemisphere (Figures 3B, C).

\section{CASE 4}

A 39-year-old female, born at 36 weeks via normal spontaneous vaginal delivery, had a normal course of development until age 1 year when she had meningitis. Since then, she had left hemiparesis and learning difficulties.
No seizure disorder was noted and the family history was unremarkable. Left-sided spastic hemiparesis and hyperreflexia were noted on physical examination. Cranial CT demonstrated a small right hemicranium and ipsilateral ventricular enlargement in the affected right hemisphere (Figures 4A, B).

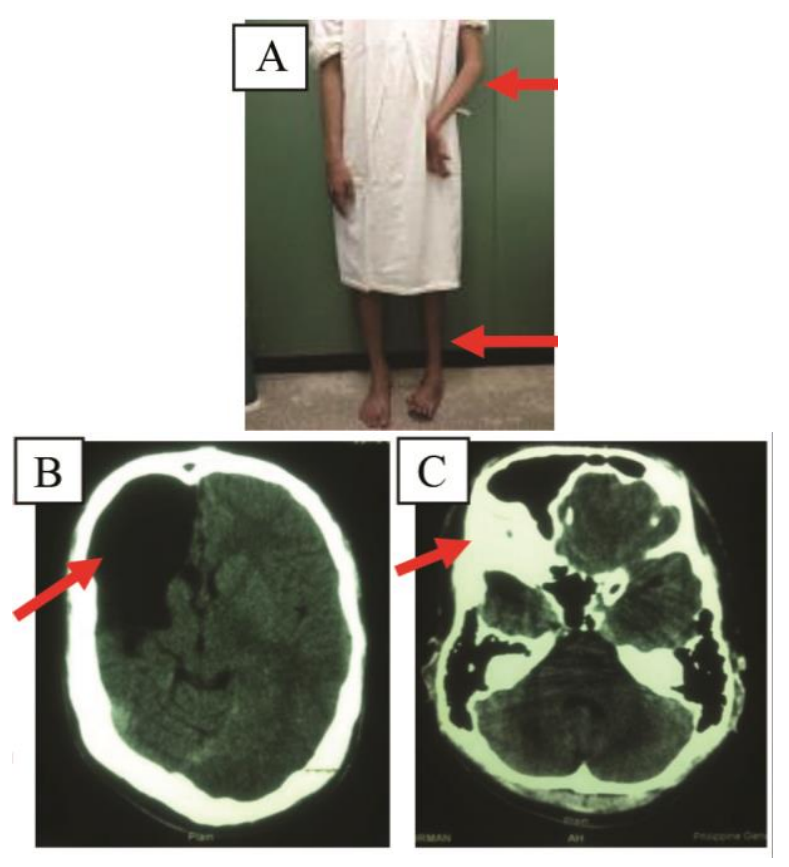

Figure 3. A. Significant atrophy of the left extremities is documented. B. Cranial CT showed a smaller right cerebral hemisphere compared to the left. A well defined CSF attenuating focus is seen in the right frontotemporoparietal region with possible communication with the dilated right lateral ventricle. C. Hyperpneumatization of the right frontal, ethmoid and sphenoid sinuses with generalized thickening of the bony calvarium.
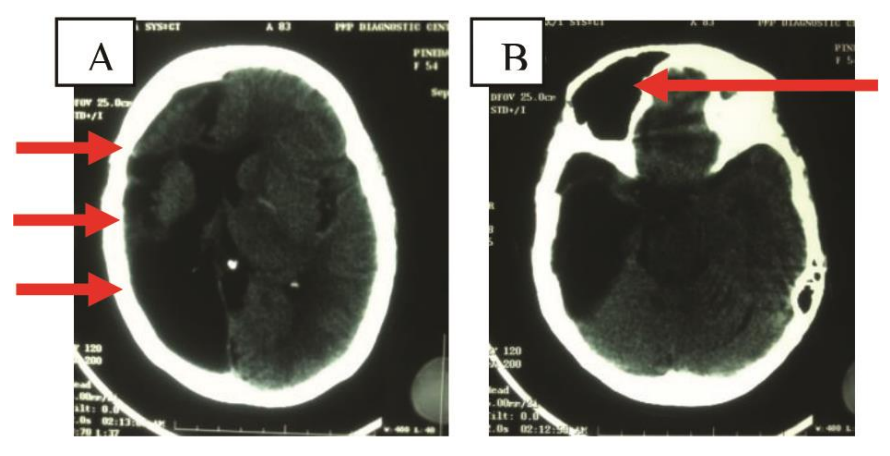

Figure 4. A. A small right hemicranium and ipsilateral ventricular enlargement in the affected minor hemisphere is seen. B. Hyperpneumatization of the right frontal sinus as well as calvarial thickening is likewise appreciated. 


\section{Discussion}

In 1933, Dyke, Davidoff, and Masson described the radiographic findings in a series of nine patients characterized clinically by hemiparesis, seizures, facial asymmetry, and mental retardation. ${ }^{1}$ The radiographic changes included calvarial thickening, elevation of the greater wing of sphenoid and petrous ridge and dilatation of the ipsilateral frontal and ethmoid sinuses. Several degrees of hemiparesis, seizures, and mental retardation can accompany this syndrome. The clinical manifestations may vary depending on the extent of brain damage. The pathogenesis of this syndrome remains controversial but it is hypothesized that ischemic episodes from a variety of different causes reduce the production of brain derived neurotrophic factors, which in turn lead to cerebral atrophy. ${ }^{2}$ It can result from a cerebral insult that may occur either in utero or early in life.

The etiology of cerebral hemiatrophy may be divided into two categories: congenital or acquired. There is usually no apparent causative factor for the congenital type. It is believed that cerebral damage that is vascular in origin most likely occurs during intrauterine life, and the signs and symptoms are present at birth or shortly thereafter. ${ }^{3,4}$ In the acquired type, cerebral insults occur during the perinatal period or later. It may have a variable time period of presentation, appearing up to and into adolescence. Possible etiological factors include infection, trauma, vascular abnormalities of the cerebral circulation, and ischemic or hemorrhagic states. Subependymal germinal matrix and intraventricular hemorrhage have also been implicated among premature infants. ${ }^{5}$ In contrast to the acquired type, the congenital type of cerebral hemiatrophy shows ipsilateral midline shift and ventricular dilatation as well compensatory cranial changes.6,7 Prominent sulcal spaces will similarly be absent because of deficient gyri and sulci formation. In a study done by Tasdemir et al., variable degrees of radiographic findings such as ipsilateral calvarial thickening, hyperpneumatization of paranasal sinuses and dilation of cerebral ventricle were reported in all five cases of congenital and acquired DDMS. Likewise, no relationship was found between these pathological changes and the time after onset of the disease. ${ }^{8}$

In the present study, hemiparesis was found in all four cases. Learning disabilities and seizures were noted in the history of three patients. Two of our cases were considered acquired DDMS. In these patients, symptoms appeared following CNS infection and perinatal trauma. In the remaining two patients, there was no known previous history of disease and these cases were considered congenital. In all the patients, variable degrees of ipsilateral calvarial thickening, hyperpneumatization of paranasal sinuses and dilatation of cerebral ventricles were seen.
Both case 1 and case 2 patients who were considered cases of congenital DDMS are siblings who presented with similar clinical findings of intractable seizures, right-sided hemiparesis and mental retardation. In these cases, there was no history of significant antenatal or perinatal disease or complications. Family medical history was significant for seizure disorder (Figure 5). In the literature, Karadamir et al. described the first case of congenital DDMS in an identical twin. In this report, the other identical twin and two other male siblings were in good health. In contrast, the third case of a 20-year-old male in this report had an identical twin that expired at birth. To date, no association between a genetic or hereditary factor and DDMS has been shown in the literature.

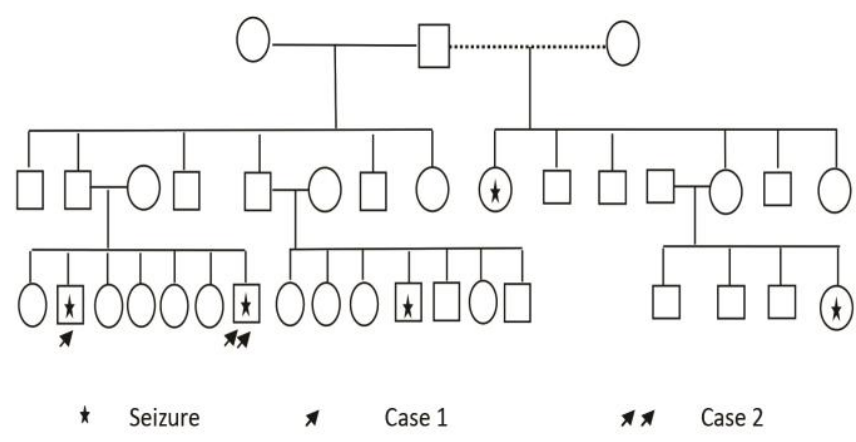

Figure 5. Family Genogram. Affected patients have several relatives diagnosed with seizure disorder.

This study is of particular interest because of the rarity of the disease not only in our setting but worldwide. Correlation between a possible genetically transmitted disease and DDMS may be an avenue for further research.

\section{Conclusion}

To the best of our knowledge, this is the first observational study of Dyke-Davidoff-Masson Syndrome reported in the Philippines. The purpose of this study is to enable early recognition of Dyke-Davidoff-Masson Syndrome. The insufficiency of case reports might have contributed to the lack of awareness among the medical community of this syndrome. In the evaluation of patients presenting with seizures, mental retardation and hemiparesis, in association with radiological features of cerebral hemiatrophy, calvarial thickening and hyperpneumatization of paranasal sinuses, a diagnosis of Dyke-Davidoff-Masson Syndrome is to be considered. A proper clinical history and characteristic neuroimaging findings provide the correct diagnosis. 


\section{References}

1. Dyke CG, Davidoff LM, Masson CB. Cerebral hemiatrophy and homolateral hypertrophy of the skull and sinuses. Surg Gynecol Obstet. 1933; 57:588-600.

2. Ono K, Komai K, Ikeda T. Dyke-Davidoff-Masson syndrome manifested by seizure in late childhood: a case report. J Clin Neurosci. 2003; 10:36771.

3. Ünal O, Caksen H, Kiymaz N, Dilek I, Kayan M, Anlar O. Cerebral hemiatrophy associated with hematological and developmental disorders. J Pediatr Neurol. 2004; 2(3):169-72.

4. Aguiar PH, Liu CW, Leitao H, et al. MR and CT imaging in the DykeDavidoff-Masson syndrome. Arq Neuropsiquiatr. 1998; 56(4):803-7.

5. Sener RN, Jinkins JR. MR of craniocerebral hemiatrophy. Clin Imaging. 1992; 16(2):93-7.

6. Atalar MH, Icagasioglu D, Tas F. Cerebral hemiatrophy (Dyke-DavidoffMasson syndrome) in childhood: Clinicoradiological analysis of 19 cases. Pediatr Int. 2007; 49:70-5.

7. Kochar DK, Jain N, Sharma BV, Kumawat BL, Meena CB. DykeDavidoff-Masson syndrome: Neuroimage. Neurol India. 2001; 49:417-8.

8. Tasdemir HA, Incesu L, Yazicioglu AK, Belet Ü, Güngör L. DykeDavidoff-Masson syndrome. Clin Imaging. 2002; 26(1):13-7.

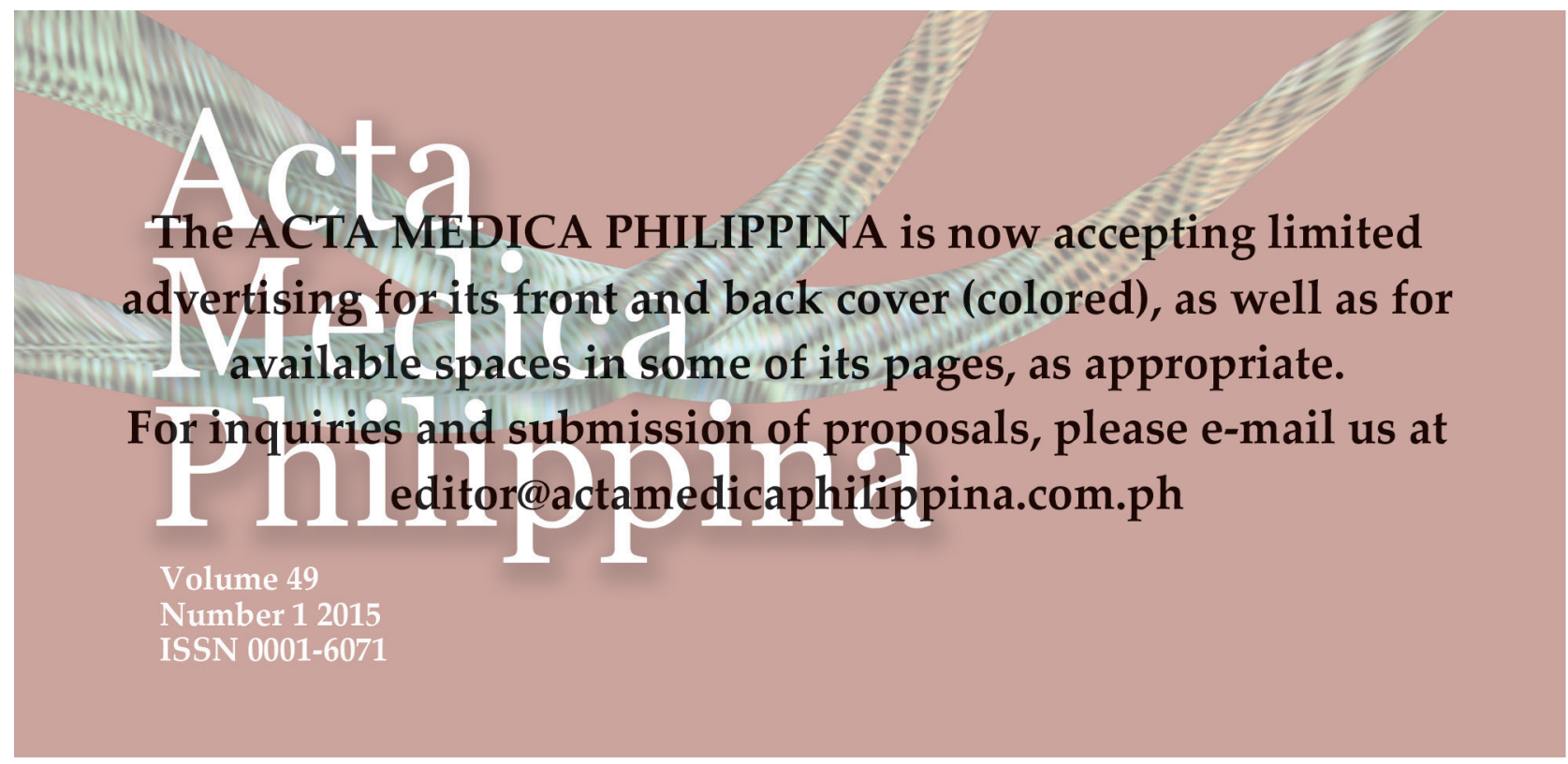

